\title{
Designing Green ECG Machine Based on Artix-7 28nm FPGA
}

\author{
${ }^{1}$ Chandrashekhar Patel, ${ }^{2}$ Puneet Chandra Verma, ${ }^{3}$ Pragya \\ Agarwal, ${ }^{4}$ Apurva Omer, ${ }^{5}$ Bhaskar Gururani, ${ }^{6}$ Sanchit Verma
}

\author{
${ }^{1,3,4}$ Department of Computer Science DSVV Haridwar, India \\ 2,5,6 Seemant institute of technology Pithoragarh India \\ 1'shekharrockin1988@gmail.com, ${ }^{2}$ cvermapuneet@gmail.com, ${ }^{3}$ pragyaagarwal30@gm \\ ail.com, ${ }^{4}$ apurva.omer@dsvv.ac.in, ${ }^{5}$ gururanibhaskar272@gmail.com, \\ ${ }^{6}$ sanchit.verma20123@gmail.com
}

\begin{abstract}
In particular this work we checked the total power consumption of ECG machine design by applying different-different techniques like frequency, voltage and ambient temperature. At first we scaled down the voltage from 2.5 volt to 1.5 volt and found that $97.46 \%$ of reduction in total power consumption. In second we scaled down our frequency form $500 \mathrm{MHz}$ to $25 \mathrm{MHz}$ and found $2.99 \%$ reduction in total power consumption. In last we scaled down our ambient temperature from $60 \mathrm{~F}$ to $20 \mathrm{~F}$ and found $25.61 \%$ of reduction in total power of consumption. We have implemented our designed on 28nm Artix7 FPGA Family.
\end{abstract}

Keywords: Thermal Aware Design, FPGA, Energy Efficient Design.

\section{Introduction}

In medical field lots of researchers are working on new medical equipment which can becomes useful to check the early detection of the disease and for routine checkup of body. Here we have designed a ECG or EKG system through FPGA technology which is helpful in observing the heart diseases. For the diagnostic system ECG is very important, also known as EKG. ECG test enclose the heart's electrical activity as shown in the figure 1. In Electrocardiography we are trying to place electrodes on the skin and try to calculate the heart activity over the period of time. So it is basically use for cardiology test. Now in this era power consumption is main concern of any electrical equipment. So we are trying to make medical equipment which will consume less power. If we design the less power consumption equipment for the medical industry then it will good for the society. In figure: 2, figure: 3 we have shown the Top Level of Schematic and RTL Schematic of ECG Machine. 


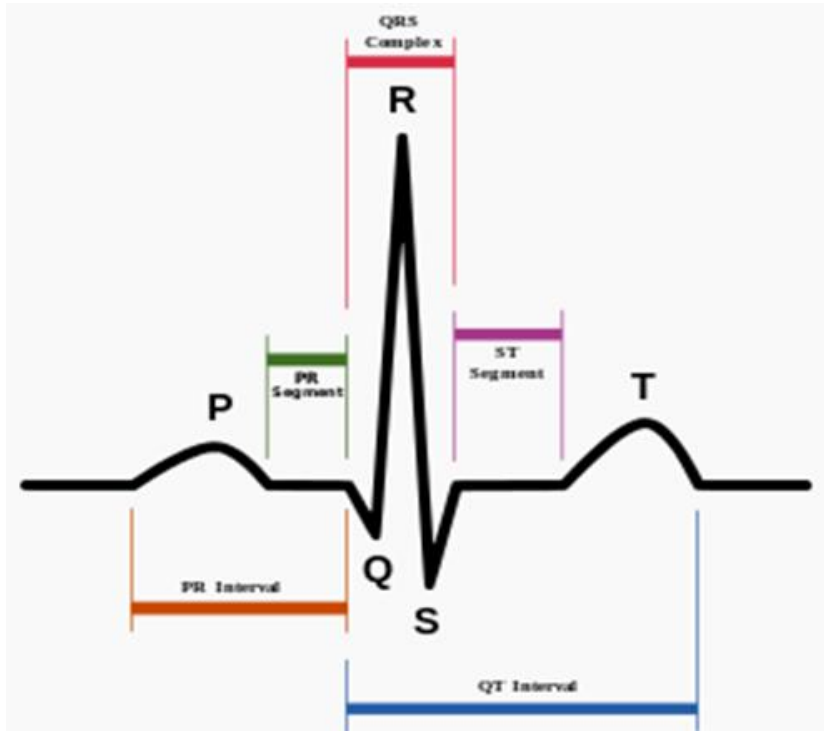

Fig. 1. ECG of a heart in normal sinus rhythm

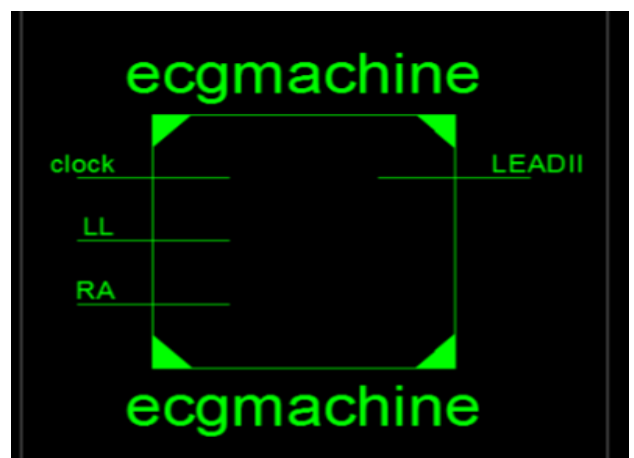

Fig. 2. Top Level of Schematic of ECG Machine

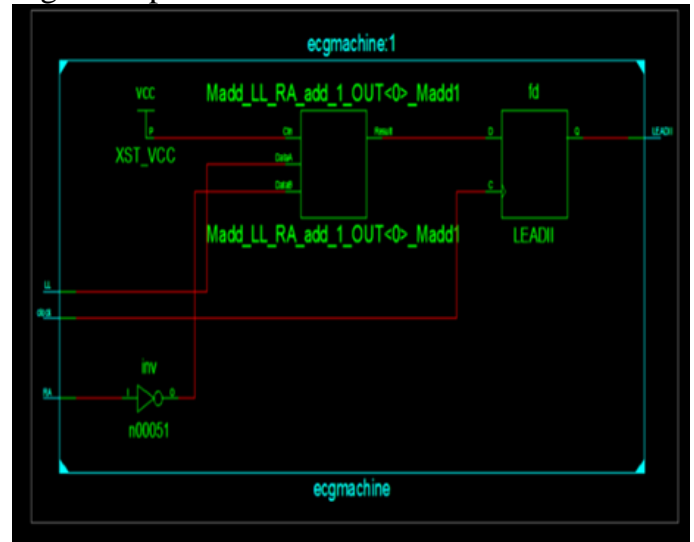

Fig. 3. RTL Schematic of ECG Machine

\section{Related Survey}

Energy Efficient Counter Design using Voltage Scaling On FPGA [1]. Green ECG Machine Design Using Different Logic Families [2]. A Biometric ECG Identification 


\section{Gyancity Journal of Engineering and Technology,}

Vol.3, No.1, pp. 36-41, January 2017

ISSN: 2456-0065 DOI: 10.21058/gjet.2017.31006

using LNF in Wireless Body Area Sensor Network [3]. Development of an embedded system and matlab based GUI for online acquisition and analysis of ECG signal [4]. Design of a Low Cost ECG system: Review [5]. Thermal Aware Low Power Universal Asynchronous Receiver Transmitter Design on FPGA [6]. Leakage Power Reduction with Various IO Standards and Dynamic Voltage Scaling in Vedic Multiplier on Virtex-6 FPGA [7]. HSTL IO Standards Based Processor Specific Green Counter [8].

\section{Power Analysis}

Table 1: Power Dissipation with variation in Voltage

\begin{tabular}{|l|l|l|l|l|l|l|}
\hline & Clock & Logic & Signals & IOs & Leakage & Total \\
\hline 1.5 Volt & 0.001 & 0.00 & 0.00 & 0.051 & 5.634 & 5.687 \\
\hline 1.8 Volt & 0.001 & 0.00 & 0.00 & 0.053 & 19.744 & 19.798 \\
\hline 2.0 Volt & 0.002 & 0.00 & 0.00 & 0.054 & 39.640 & 39.696 \\
\hline 2.2 Volt & 0.002 & 0.00 & 0.00 & 0.055 & 79.498 & 79.555 \\
\hline 2.5 Volt & 0.002 & 0.00 & 0.00 & 0.056 & 224.048 & 224.107 \\
\hline
\end{tabular}

In the table 1 we have calculated our total power consumption at $1.5 \mathrm{~V}, 1.8 \mathrm{~V}, 2.0 \mathrm{~V}$, $2.2 \mathrm{~V}$ and $2.5 \mathrm{~V}$ device operating voltage respectively and found that when we scaled down the voltage form 2.5 volt to 1.5 volt then we got $97.46 \%$ of reduction in total power. We found that there is very tiny change in the clock power. There is no change value for the Logic and Signals. We have also shown our analysis work through the bar graph as shown in the figure 4 .

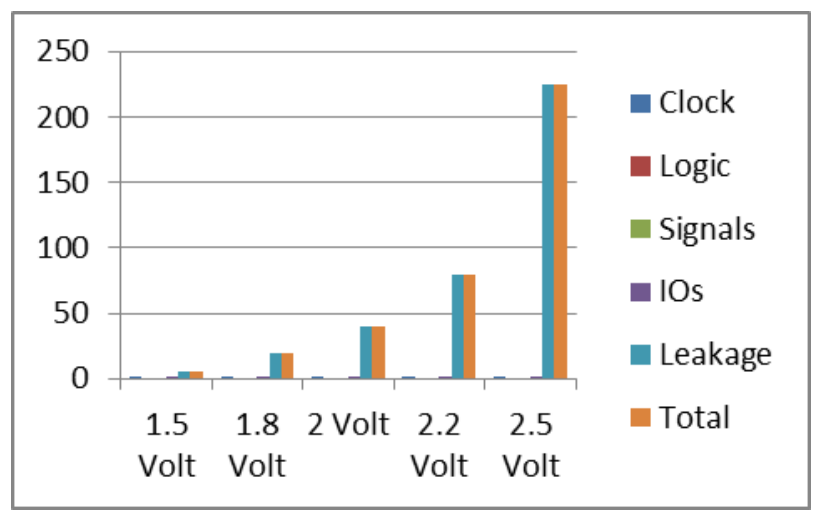

Fig. 4. Power consumption with variation in Voltage 
Table 2: Power Dissipation with variation in Frequency

\begin{tabular}{|l|l|l|l|l|l|l|}
\hline & Clock & Logic & Signal & IOs & Leakage & Total \\
\hline $25 \mathrm{MHZ}$ & 0.001 & 0.00 & 0.00 & 0.000 & 1.293 & 1.294 \\
\hline $200 \mathrm{MHZ}$ & 0.002 & 0.00 & 0.00 & 0.000 & 1.293 & 1.295 \\
\hline $300 \mathrm{MHZ}$ & 0.009 & 0.00 & 0.00 & 0.009 & 1.293 & 1.312 \\
\hline $400 \mathrm{MHZ}$ & 0.012 & 0.00 & 0.00 & 0.016 & 1.293 & 1.322 \\
\hline $500 \mathrm{MHZ}$ & 0.015 & 0.00 & 0.00 & 0.025 & 1.294 & 1.334 \\
\hline
\end{tabular}

In the table 2 we have applied frequency scaling technique where we use $(25 \mathrm{MHz}$, $200 \mathrm{MHz}, 300 \mathrm{MHz}, 400 \mathrm{MHz}$, and $500 \mathrm{MHz}$ ) following frequency rages and found when we scaled down the frequency from $500 \mathrm{MHz}$ to $25 \mathrm{MHz}$ then we got $2.99 \%$ of reduction in total power consumption. We also found there is no change in logic and signal power and small changes in clock power. We have also done this analysis by the bar graph as shown in the figure 5.

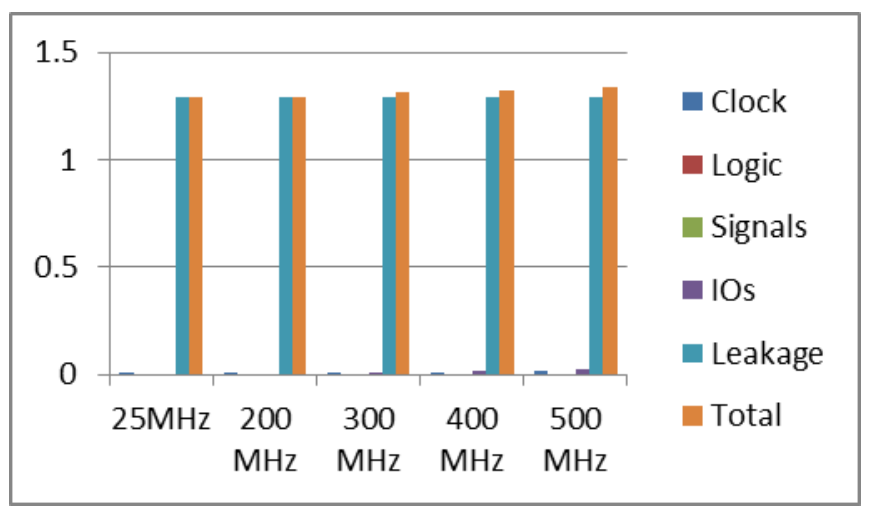

Fig. 5 Power Consumption with variation in Frequency

Table 3: Power Dissipation with variation in Ambient Temperature

\begin{tabular}{|c|c|c|c|c|c|c|}
\hline & Clock & Logic & Signal & IOs & Leakage & Total \\
\hline $20 \mathrm{~F}$ & 0.015 & 0.00 & 0.00 & 0.025 & 1.092 & 1.132 \\
\hline $30 \mathrm{~F}$ & 0.015 & 0.00 & 0.00 & 0.025 & 1.150 & 1.191 \\
\hline $40 \mathrm{~F}$ & 0.015 & 0.00 & 0.00 & 0.025 & 1.217 & 1.258 \\
\hline $50 \mathrm{~F}$ & 0.015 & 0.00 & 0.00 & 0.025 & 1.294 & 1.334 \\
\hline $60 \mathrm{~F}$ & 0.015 & 0.00 & 0.00 & 0.025 & 1.381 & 1.422 \\
\hline
\end{tabular}

In Table 3 we calculated total power consumption by applying the ambient temperature techniques. We use $(20 \mathrm{~F}, 30 \mathrm{~F}, 40 \mathrm{~F}$. $50 \mathrm{~F}, 60 \mathrm{~F})$ following temperature ranges and found when we reduced the temperature from $60 \mathrm{~F}$ to $20 \mathrm{~F}$ then we got $25.61 \%$ reduction in total power consumption. We also converted this same analysis through bar graph for better understanding as shown in figure 6 . 


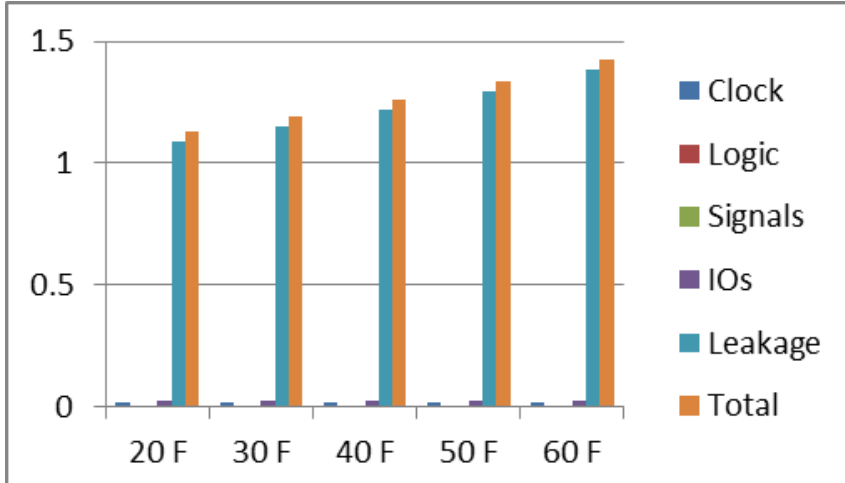

Fig. 6: Power Consumption with variation in Ambient Temperature

\section{Conclusion}

In this work we have implemented first voltage scaling techniques and found that when we scaled down the frequency from 2.5 volt to 1.5 volt then we reduced the power consumption by $97.46 \%$.Secondly we implemented frequency scaling techniques and found that when we scaled down the frequency from $500 \mathrm{MHz}$ to 25 $\mathrm{MHz}$ then we reduced the power consumption by the $2.99 \%$.In last we applied ambient temperature techniques and found that when we scaled down the temperature from $60 \mathrm{~F}$ to $20 \mathrm{~F}$ then we got $25.61 \%$ of reduction in power consumption.

\section{Future Scope}

In this work, ECG Design is implemented on $28 \mathrm{~nm}$ on Airtex-7, but we have a scope to redesign this ECG machine on latest $65 \mathrm{~nm}$ Virtex-5 and 40nm Virtex-6, 90nm Virtex-4 FPGA to make the most energy efficient ECG design. We can also take more frequency range to redesign energy efficient ECG design

\section{Acknowledgment}

The author would like to acknowledge Gyancity Research Lab (India \& Overseas) for their valuable support for completing this research paper.

\section{References}

[1] T.Gupta,G.Verma, A.Kaur, B.pandey, A.Singh, and T.Kaur. "Energy Efficient Counter Design using Voltage Scaling On FPGA" In Communication System and Networks Technologies (CSNT), 2015 Fifth International Conference On pp.816-819. IEEE, 2015.

[2] S.Aggarwal, G.Verma, R.Kumar, A.Kaur, B.Pandey, S.Singh "Green ECG Machine Design Using Different Logic Families" In CommunicationSystem and Networks Technologies (CSNT), 2015 Fifth International Conference 978-1-4799-1797-6/15 IEEE, 2015.

[3] L.Yogita , H.Pankaj. Rangaree, "A Biometric ECG Identification using LNF in Wireless Body Area Sensor Network”, International Journal of Recent Technology and Engineering (IJRTE), ISSN:2277-3878, Volume-1, Issue-2, ( June 2012)

[4] R. Gupta, J N .Bera ,M. Mitra, "Development of an embedded system and matlab based GUI for online acquisition and analysis of ECGsignal",Measurement 43(2010 Science Direct, pp 1119-1126,(2010). 


\section{Gyancity Journal of Engineering and Technology, Vol.3, No.1, pp. 36-41, January 2017 ISSN: 2456-0065 DOI: 10.21058/gjet.2017.31006}

[5] K. Krishna bai, S. C. Prasanna Kumar, "Design of a Low Cost ECG system: Review", Canadian Journal on Biomedical Engineering\& Technology, Vol. 3, No. 2, February 2012.

[6] S.Singh, A.Jain. A.Kaur, B.Pandey, "Thermal Aware Low Power UniversalAsynchronous Receiver Transmitter Design on FPGA", IEEE 6th International Conference on Computational Intelligence and Communication Networks (CICN), Bhopal, 14-16 November,2014.

[7] B.Pandey, Md. Rahman, A.Saxena, A.Hussain, B.Das "Leakage Power Reduction with Various IO Standards and Dynamic Voltage Scaling in Vedic Multiplier on Virtex-6 FPGA". In Indian Journal of Science and Technology, Vol 9(25),July 2016.

[8] A.Saxena, A.Bhatt B.Pandey, P.Tripathi"HSTL IO Standards Based Processor Specific Green Counter.'In International Journal of Control and Automation, Vol. 9, No. 7, (2016), pp. 331342 